\title{
Centimeter-Sized Single-Orientation Monolayer Hexagonal Boron Nitride With or Without Nanovoids
}

\author{
Huanyao Cun, ${ }^{*}+, \S \odot$ Adrian Hemmi, ${ }^{\dagger}$ Elisa Miniussi, ${ }^{\dagger}$ Carlo Bernard, ${ }^{\dagger}$ Benjamin Probst, ${ }^{\ddagger}$ Ke Liu, ${ }^{\S}$ \\ Duncan T. L. Alexander, ${ }^{\| \odot}$ Armin Kleibert, ${ }^{\perp}$ Gerson Mette, ${ }^{\dagger, \# \odot ~ M i c h a e l ~ W e i n l, ~}{ }^{\nabla}$ Matthias Schreck, ${ }^{\nabla}$ \\ Jürg Osterwalder, ${ }^{\dagger}$ Aleksandra Radenovic, ${ }^{\S \subseteq}$ and Thomas Greber ${ }^{\dagger}$ \\ ${ }^{\dagger}$ Physik-Institut and ${ }^{\ddagger}$ Department of Chemistry, Universität Zürich, 8057 Zürich, Switzerland \\ ${ }^{\S}$ Institute of Bioengineering and "Centre Interdisciplinaire de Microscopie Électronique, EPFL, 1015 Lausanne, Switzerland \\ ${ }^{\perp}$ Swiss Light Source, Paul Scherrer Institut, 5232 Villigen, Switzerland \\ ${ }^{\#}$ Fachbereich Physik, Philipps-Universität Marburg, 35037 Marburg, Germany \\ $\nabla$ Institut für Physik, Universität Augsburg, 86159 Augsburg, Germany
}

\section{Supporting Information}

ABSTRACT: Large-area hexagonal boron nitride ( $h$-BN) promises many new applications of two-dimensional materials, such as the protective packing of reactive surfaces or as membranes in liquids. However, scalable production beyond exfoliation from bulk single crystals remained a major challenge. Single-orientation monolayer $h$-BN nanomesh is grown on 4 in. wafer single crystalline rhodium films and transferred on arbitrary substrates such as $\mathrm{SiO}_{2}$, germanium, or transmission electron microscopy grids. The transfer process involves application of tetraoctylammonium bromide before
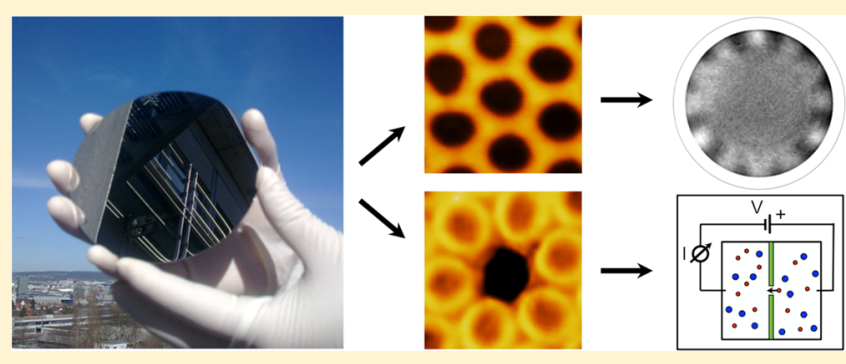
electrochemical hydrogen delamination. The material performance is demonstrated with two applications. First, protective sealing of $h$ - $\mathrm{BN}$ is shown by preserving germanium from oxidation in air at high temperatures. Second, the membrane functionality of the single $h$-BN layer is demonstrated in aqueous solutions. Here, we employ a growth substrate intrinsic preparation scheme to create regular $2 \mathrm{~nm}$ holes that serve as ion channels in liquids.

KEYWORDS: Hexagonal boron nitride, 2D materials, chemical vapor deposition, nanoporous membranes, electrochemical delamination

$\mathrm{T}$ wo-dimensional (2D) materials are expected to significantly widen the prospects for condensed matter systems ${ }^{1}$ and to unlock new functionalities in optoelectronics ${ }^{2}$ or ionic transport. $^{3-5}$ However, if the materials shall become useful, they have to be produced in a scalable way. This means that the exfoliation procedure of $2 \mathrm{D}$ flakes from $3 \mathrm{D}$ single crystals has to be replaced by processes that yield large-area material. This also applies to hexagonal boron nitride $(h-\mathrm{BN})$ that emerges to be ideal for the encapsulation of graphene-based electronic devices $^{6}$ or that is a promising membrane material in liquids., Previous reports on transfer of chemical vapor deposition (CVD) $h$-BN by the $\mathrm{H}_{2}$ "bubbling" delamination method ${ }^{7-9}$ show that the transfer size was limited to micrometer flakes. ${ }^{7}$ Recent work reports the transfer of large-area multilayer $h$-BN via the etching of the sapphire substrates. ${ }^{8} \mathrm{Up}$ to now, the transfer of large-area $h$-BN from metal growth substrates remained an unsolved problem. Here, we report on the transfer process of large-area single layer $h$-BN with single orientation that is grown on 4 in. wafer-scale single-crystalline $\mathrm{Rh}(111)$ films. The transfer of continuous layers is evidenced for the bare eye with oxidation protection experiments of germanium. The two-step transfer protocol involves electrochemical tetraoctylammonium ion $\left(\mathrm{TOA}^{+}\right)$treatment prior to a hydrogen "bubbling" at the interface of $h$-BN and metal substrates. It is reliable (high transfer rates above 90\%) and gentle because the transferred $h$-BN layers not only maintain single crystal orientation but also preserve surface modifications such as $2 \mathrm{~nm}$ voids that rely on the growth substrates.

As a growth substrate, we employ twin-free single-crystalline $\mathrm{Rh}(111)$ thin films on $4 \mathrm{in}$. $\mathrm{Si}(111)$ wafers. A corrugated $h$-BN layer is grown in ultrahigh vacuum (UHV) by CVD. ${ }^{10,11}$ The corrugation appears as a "mesh" superstructure with "pores" of about $2 \mathrm{~nm}$ diameter, in which the $\mathrm{BN}$ is closely attached to the substrate, and a surrounding "wire" region with a weaker bonding and a larger layer-substrate distance (Figure 1a). ${ }^{12-14}$ This nanomesh surface is stable in air and in liquids, ${ }^{13,15}$ and its corrugation can be switched on and off via hydrogen intercalation, either in vacuum ${ }^{16}$ or in liquids. ${ }^{4}$ With additional preparation steps, single $2 \mathrm{~nm}$ voids are fabricated with the

Received: November 9, 2017

Revised: January 1, 2018

Published: January 9, 2018 

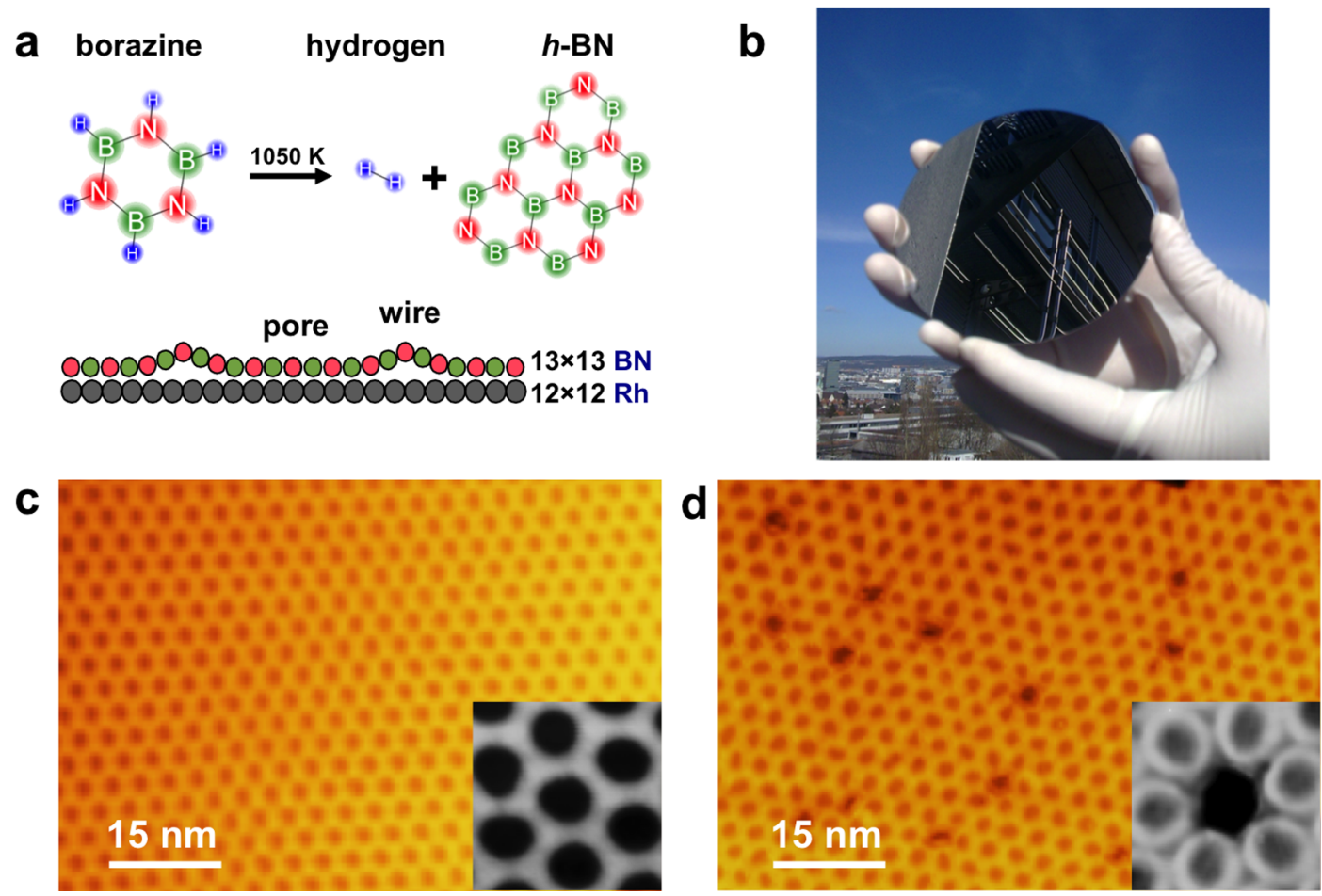

Figure 1. $h$-BN nanomesh on $\mathrm{Rh}(111)$ with and without $2 \mathrm{~nm}$ voids. (a) Chemical structure of the borazine $(\mathrm{HBNH})_{3}$ precursor and its decomposition to form a $h$-BN monolayer and $\mathrm{H}_{2}$ gas on a hot $\mathrm{Rh}(111)$ surface. The $h$-BN corrugation is caused by the lattice mismatch that accommodates $13 \times 13 \mathrm{BN}$ on $12 \times 12 \mathrm{Rh}$ units. (b) Photograph of a 4 in. wafer single-crystalline $\mathrm{Rh}(111)$ thin film substrate in Zürich air, on which the nanomesh is grown. (c) Room-temperature STM images of pristine $h-\mathrm{BN}$ nanomesh on $\mathrm{Rh}(111), U_{\mathrm{t}}=-1.20 \mathrm{~V}$, and $I_{\mathrm{t}}=0.50 \mathrm{nA}$. (d) Multiple $2 \mathrm{~nm}$ voids at pore sites generated by the "can-opener" effect after annealing of $h-\mathrm{BN} / \mathrm{Ar} / \mathrm{Rh}(111)$ to $900 \mathrm{~K}, U_{\mathrm{t}}=-1.20 \mathrm{~V}, I_{\mathrm{t}}=0.50 \mathrm{nA}$. The two right-bottom insets show (c) $9 \mathrm{~nm} \times 9 \mathrm{~nm}$ zoomed-in images of pristine $h$-BN nanomesh and (d) voidal $v$-BN.

"can-opener" effect to form a surface with uniformly distributed voids in the $h$-BN layer, ${ }^{17,18}$ termed voidal boron nitride $(v$ $\mathrm{BN})$. The functionality of the freestanding $v$-BN material is demonstrated by immersion of such nanoporous membranes in aqueous solutions and the measurement of ion conductivity.

Results and Discussion. Pristine $h$-BN monolayers are prepared by the high-temperature CVD method with borazine $(\mathrm{HBNH})_{3}$ as precursor on single-crystalline $\mathrm{Rh}(111) /$ yttriastabilized zirconia(YSZ)/ $\mathrm{Si}(111)$ substrates at $1050 \mathrm{~K}{ }^{11,12}$ The sample fabrication is scaled up to 4 in. wafers (Figure $1 \mathrm{~b}$ ), and the quality of the $h$-BN is comparable to the one fabricated on $\mathrm{Rh}(111)$ single crystals (Figure $1 \mathrm{c}) .{ }^{11}$ The $v$-BN is prepared by exposing $h$-BN/Rh(111) to low-energy $\mathrm{Ar}$ ions followed by a subsequent annealing procedure. ${ }^{17,19}$ The ion bombardment generates vacancy defects in the boron nitride layer, which, upon annealing to $900 \mathrm{~K}$, leads to the "can-opener" effect, i.e., the formation of $2 \mathrm{~nm}$ voids in the $h$-BN layer, ${ }^{18}$ as shown in the scanning tunneling microscopy (STM) image in Figure 1d.

Figure 2 is a schematic illustration of the transfer procedure for single layer $h$-BN. After UHV-CVD growth (Figure 2-1), the $h$-BN samples are introduced into a three-electrode setup in a tetraoctylammonium bromide ( $\mathrm{TOABr}$ )/acetonitrile solution (Figure 2-2). ${ }^{20}$ The reference potential is kept at $-1.9 \mathrm{~V}$ for a thorough diffusion of molecules and ions in the solution and for enabling chemical reactions on the electrodes. This $\mathrm{TOA}^{+}$treatment step ensures transfer rates above 90\%, while its omission typically yields transfer rates below $10 \%$, as shown in the Figure S1. Subsequently, the TOA-treated sample (TOA $/ h$ $\mathrm{BN} / \mathrm{Rh}$ ) is spin-coated with a polymethylmetacrylate (PMMA) film (Figure 2-3), followed by electrochemical hydrogen intercalation $^{4}$ and subsequent "bubbling" delamination ${ }^{21,22}$
(Figure 2-4). Finally, the delaminated PMMA/TOA/ $h-\mathrm{BN}$ film in Figure 2-5 may be transferred to an arbitrary substrate.

After transfer, the remaining $\mathrm{Rh}$ substrates, as indicated in Figure 2-6, can be recycled to regrow $h-\mathrm{BN}$, in which the quality of the regrown $h$-BN monolayer stays high (Figure S2). In the following, we report transfer results on transmission electron microscopy (TEM) grids (Figure 2-7), on $\mathrm{SiO}_{2}$ and on $\mathrm{Ge}(111)$ wafers (Figure 2-8).

To characterize the transferred PMMA/TOA/ $h$-BN layer, they are placed onto $\mathrm{SiO}_{2} / \mathrm{Si}$ substrates and annealed in vacuum to $650 \mathrm{~K}$. The thickness of the $80 \mathrm{~nm} \mathrm{SiO}{ }_{2}$ is chosen to maximize the optical contrast of $h-\mathrm{BN}^{23}$ and to still allow good electron spectroscopy results by avoiding strong sample charging. Figure $3 \mathrm{a}, \mathrm{b}$ shows the X-ray photoelectron spectroscopy (XPS) spectra of B1s and N1s before (black) and after (red) transfer (the survey spectra are shown in Figure S3). We define the transfer rate as the ratio of the XPS intensities of transferred $\mathrm{BN}$ and pristine $\mathrm{BN}$ (B1s and N1s) (see section 4 in the Supporting Information). The XPS results indicate that $95 \%$ of the $h$-BN monolayer is transferred, which fits well with the optical microscopy observation of intact layers (Figure S5). The angle scanned X-ray photoelectron diffraction (XPD) of $\mathrm{B} 1 \mathrm{~s}$ and N1s in Figure 3c,e indicate a flat single-orientation $h$ $\mathrm{BN}$ monolayer transferred onto amorphous $\mathrm{SiO}_{2}$. The 3-fold symmetry with the 3 grazing angle scattering intensity maxima that are rotated by 60 degree between nitrogen and boron is known from $h$-BN/Ni(111) $)^{24}$ and $h$-BN/Rh(111). ${ }^{25}$ Comparison of the XPD patterns of B1s and N1s with the atomic structure of $h$-BN in Figure $3 \mathrm{~d}$ thus proves that the transferred layer consists of large-area single-orientation $h$-BN. The monolayer $h-\mathrm{BN}$ is consistent with Raman measurements, i.e., 

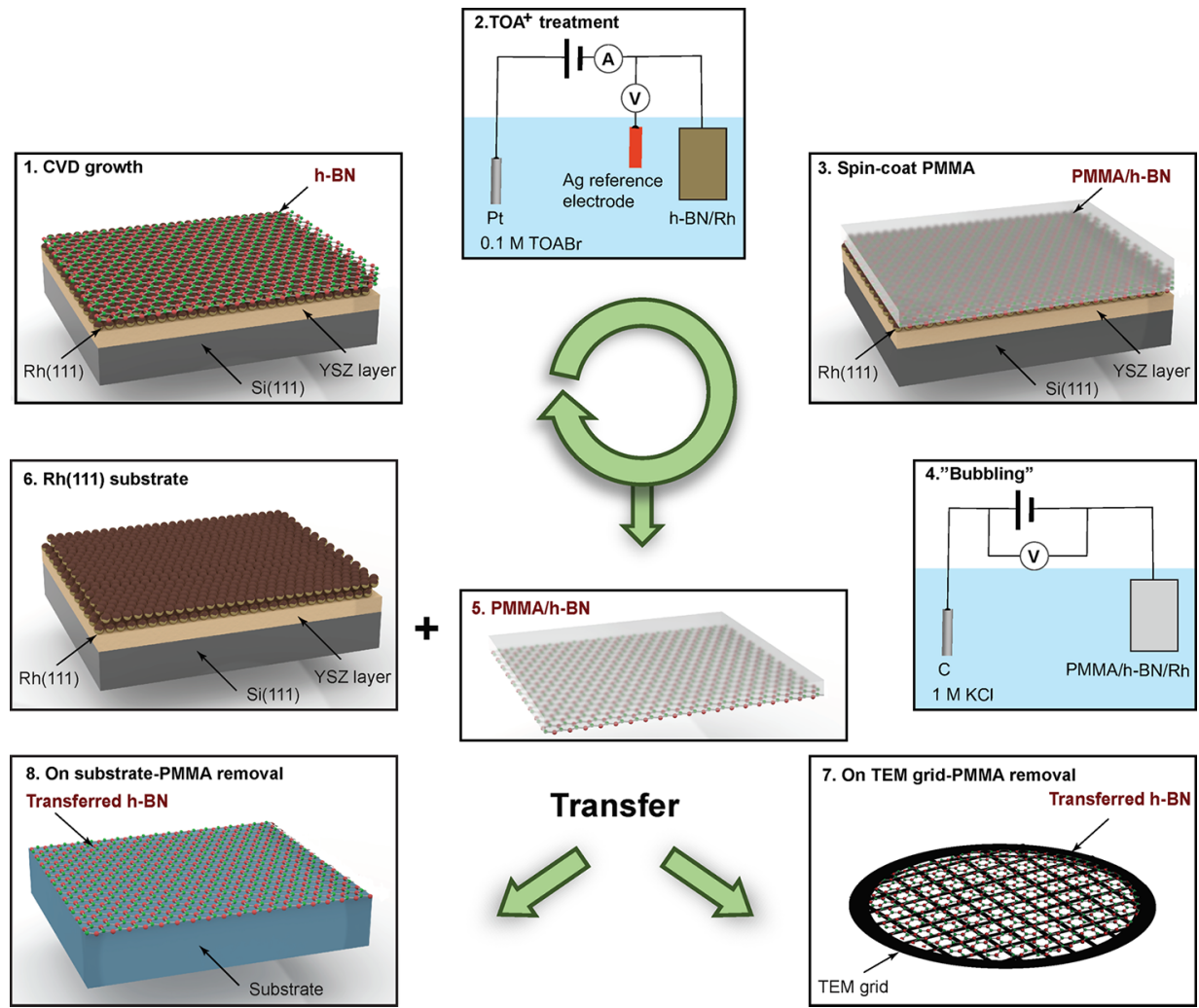

Figure 2. Two-step $\mathrm{TOA}^{+}$-assisted electrochemical transfer process of $h$-BN. (1) Structure of $h$-BN/Rh(111) film sample. On a Si(111) wafer, an yttria-stabilized zirconia layer (YSZ) $(80 \mathrm{~nm}), \mathrm{Rh}(111)(150 \mathrm{~nm})$, and a $h$-BN monolayer (with or without nanovoids) are grown. (2) First step of the transfer procedure: $\mathrm{TOA}^{+}$-treatment in a three-electrode setup, consisting of a $h-\mathrm{BN} / \mathrm{Rh}$ sample as working electrode, a $\mathrm{Pt}$ wire as counter electrode, and an $\mathrm{Ag}$ wire as reference electrode in $0.1 \mathrm{M} \mathrm{TOABr} /$ acetonitrile solution. (3) The TOA/ $h-\mathrm{BN} / \mathrm{Rh}$ sample is spin-coated with PMMA. (4) The second step includes a two-electrode setup with the PMMA/TOA/h-BN/Rh sample as working electrode and a graphite rod as counter electrode in a $1.0 \mathrm{M} \mathrm{KCl}$ solution. $\mathrm{H}_{2}$ bubble evolution between $h$-BN and $\mathrm{Rh}$ substrate lifts the PMMA/TOA/h-BN monolayer (shown in panel 5). (6) $\mathrm{Rh}(111)$ substrate after $h$-BN transfer can be used for another cycle of $h$-BN growth (panel 1 ). The PMMA/h-BN films are transferred on TEM grids (panel 7) and on $\mathrm{SiO}_{2} / \mathrm{Si}$ or Ge substrates (panel 8), followed by PMMA removal.

the characteristic Raman peak at $1368 \mathrm{~cm}^{-1}$ with a full width at half-maximum (fwhm) of $20 \mathrm{~cm}^{-1}$ (Figure S6). ${ }^{23}$

To demonstrate the protection functionality of the transferred $h$-BN, we developed a simple "litmus" test that uses the oxidation of germanium in air as an indicator. Germanium is known to oxidize at temperatures above $370{ }^{\circ} \mathrm{C}$ in air, ${ }^{26}$ where it changes color from gray to light blue. In Figure 4, the colorchange of a piece of $\mathrm{Ge}(111)$ wafer is shown during annealing from room temperature up to $610^{\circ} \mathrm{C}$. We track 3 different $2 \times$ $2 \mathrm{~mm}^{2}$ regions, which are (i) pristine Ge, (ii) covered with PMMA, and (iii) with PMMA/TOA/ $h$-BN exfoliate that is folded to a double layer. The single fold minimizes the coincidence of defects in the single layer and thus enhance the quality of protection.

After sublimation of the PMMA at about $350{ }^{\circ} \mathrm{C}$, the color is the same for all 3 spots. Upon further temperature increases, the optically visible oxidation process begins. However, the rates of color change are significantly different for the 3 regions, where it becomes clear that the $h$-BN covered spot resists oxidation up to $610{ }^{\circ} \mathrm{C}$. It is also observed that the PMMAcovered Ge area keeps the memory of having been covered, even though PMMA is no longer optically visible between 350 and $500{ }^{\circ} \mathrm{C}$. This test reflects the superior sealing behavior of atomically thin $h$-BN in extreme environments. Such membranes, for example, allow the design of packing electronic components with tightest space constraints and best thermal energy dissipation properties. Furthermore, the Ge protection test signifies a viable low-cost tool for the characterization of the quality of macroscopic BN exfoliates. In particular, we point out that the test reveals sample inhomogeneities, therefore minimizing the need for analysis with expensive surface science methods. As such, it appears to be a useful benchmark method for optimization and comparison of material production and transfer processes.

Finally, we demonstrate the application of freestanding $h$-BN and $v$-BN exfoliates in membrane applications. State of the art TEM and scanning TEM (STEM) are known for their capability to measure $h$-BN and graphene with atomic resolution. $^{27}$ The $h$-BN layers are suspended over TEM grids ( $\mathrm{Cu}$ quantifoil and $\mathrm{SiN}_{x}$ membranes) with diameters of the holes from $1.0 \mu \mathrm{m}$ down to self-made $50 \mathrm{~nm}$. Figure 5 shows spherical aberration-corrected TEM and STEM images, acquired at room temperature and with electron energies of $80 \mathrm{keV}$, which is between the boron and the nitrogen knock-on threshold in monolayer $h$-BN. ${ }^{28,29}$ Figure 5a depicts a TEM grid with $1 \mu \mathrm{m}$ holes covered by a single layer $h$-BN (dark-field TEM). The further zoom-in TEM image in Figure $5 b$ shows freestanding monolayer $h$-BN with remaining PMMA and TOA traces. Atomically clean single layer $h$-BN patches can easily be found (Figure 5c). Figure 5d-f demonstrates the application of single layer $v$-BN with $2 \mathrm{~nm}$ voids as permeable membranes in a potassium chloride $(\mathrm{KCl})$ solution. Such membranes are potential candidates for blue energy harvesting like osmotic 
a

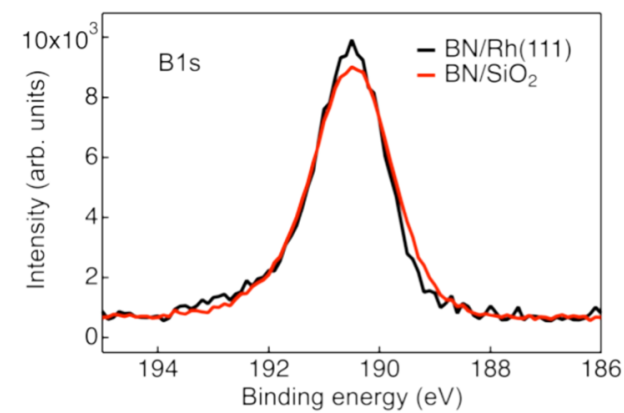

b

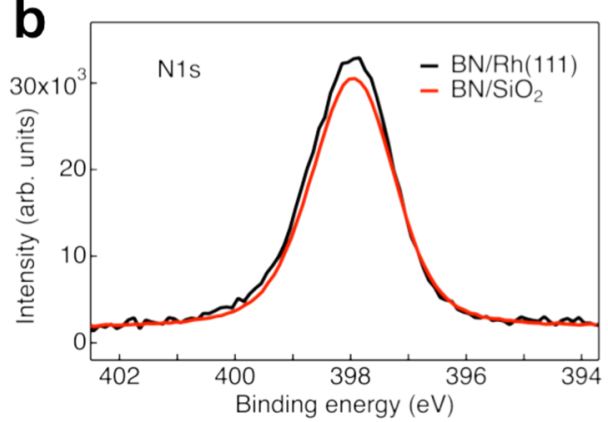

e

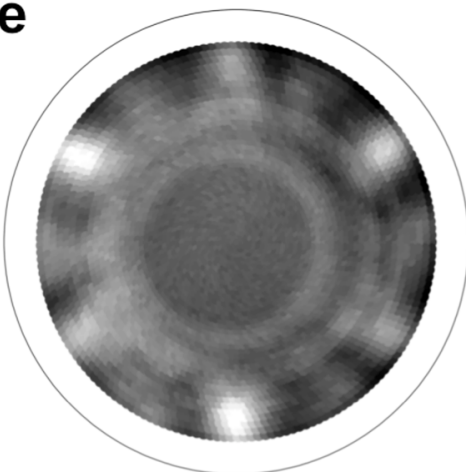

Figure 3. Mg K $\alpha$ XPS and XPD $(\hbar \omega=1253.6 \mathrm{eV})$ of $h$-BN before and after TOA ${ }^{+}$-assisted transfer. $(\mathrm{a}, \mathrm{b})$ XPS of B1s and N1s core levels on Rh before (black) and after transfer on $80 \mathrm{~nm} \mathrm{SiO}_{2} / \mathrm{Si}$ (red). The transfer rate is above $95 \%$. (c, e) X-ray photoelectron diffraction patterns of (c) B 1s and (e) $\mathrm{N}$ ls of transferred $h$-BN layer on amorphous $\mathrm{SiO}_{2}$. The 3 -fold symmetry indicates a single orientation of the $h$-BN lattice as depicted in panel d.

power generation as recently demonstrated for $\mathrm{MoS}_{2}$ membranes ${ }^{5}$ or may be used for ion separation. ${ }^{30}$

In our ion conductivity setup, ${ }^{31}$ a circular $50 \mathrm{~nm}$ hole in $20 \mathrm{~nm}$ thick SiN membrane is covered with $v$-BN membrane (Figure 5d). In Figure 5e, a $5 \mathrm{~nm} \times 5 \mathrm{~nm}$ STEM image with one void is shown. Measurements of current versus voltage $(I-V)$ characteristics demonstrate that ion conductivity across such voidal membranes can be measured. In Figure 5f, conductivities of $0.01 \mathrm{M} \mathrm{KCl}, \mathrm{pH}=8$, at room temperature are displayed for four different $v$-BN membranes. Below cell voltages of $\pm 200 \mathrm{mV}$, conductivities of $(-1 \pm 3) \times 10^{-3}, 0.48$ $\pm 0.07,0.36 \pm 0.18$, and $4.24 \pm 0.07 \mathrm{nS}$ are obtained (for details, see part 7 in the Supporting Information). ${ }^{32-34}$ From the conductance of the $\mathrm{KCl}$ solution and the void dimensions, we estimate the number of voids or ion channels to be $0,2,1$, and 16 for the 4-membrane $I-V$ curves shown in Figure 5f. The hole quantities are in the same order of magnitude as expected from the nominal $v$-BN void density before transfer of $2.3 \times$ $10^{-3} \mathrm{~nm}^{-2}$ or 3 to 6 voids on a membrane with diameter of 50 $\mathrm{nm}$. Given the uncertainties in relating the measured conductance to the void density we consider the conductivities of membrane samples (\#1 and \#2 in Figure 5f) to be close to the expectation. At variance to solid-state electrolyte boron nitride membrane applications, ${ }^{35}$ we also find ion tight membranes (\#0). \#3 with $4 \mathrm{nS}$ conductivity likely corresponds to a broken membrane.

Conclusions. A method for transfer of centimeter-sized single-orientation $h$-BN with or without surface modifications (nanovoids) from single-crystalline $\mathrm{Rh}(111)$ thin films is presented. The process involves two steps, a $\mathrm{TOA}^{+}$-treatment of the $h$-BN surface, followed by hydrogen driven delamination.
Advantages of this nondestructive isolation of CVD-grown $h$-BN include: (1) scalability; (2) substrate recycling; and (3) the fact that functionalizations that rely on the metal substrate like the "can-opener" effect are sustained. To confirm the application potential of these 2D layers, we demonstrate their thermal oxidation protection capability in air and the use as monatomic membranes with and without ion channels in aqueous solutions.

Methods. Instrumentation for Sample Fabrication. The CVD growth of $h$-BN was performed in an UHV system with a base pressure of $1 \times 10^{-10} \mathrm{mbar}$. For the high-temperature CVD fabrication of the $h$-BN nanomesh, we employed a standard procedure on $150 \mathrm{~nm}$ single-crystalline $\mathrm{Rh}(111) /$ YSZ/Si films at 4 in. wafer scale. ${ }^{11,12}$ For the $2 \mathrm{~nm}$ void generation, we used a Specs IQP 10/35 Penning-type ion source run at lowest acceleration potential. The integrated sputter charge density was $250 \mathrm{nC} / \mathrm{cm}^{2}$.

Two-Step TOA ${ }^{+}$-Assisted Electrochemical Process. The first step involves a three-electrode electrochemical setup. It consists of a $h$-BN/Rh sample as working electrode, a Pt wire as counter electrode, and an Ag-wire as reference electrode in Ar degassed $0.1 \mathrm{M} \mathrm{TOABr} /$ acetonitrile. The reference potential versus Agwire was kept at $-1.9 \mathrm{~V}$ for $10 \mathrm{~min}$, followed by a potential of $-0.2 \mathrm{~V}$ versus Ag-wire for $20 \mathrm{~s}$. Subsequently, the sample was rinsed with acetonitrile and spin-coated with 4 wt \% PMMA $(495 \mathrm{~K})$. The second step involves the PMMA/TOA/ $h-\mathrm{BN} / \mathrm{Rh}$ sample as working electrode and a graphite rod as counter electrode in $1.0 \mathrm{M} \mathrm{KCl}$ solution. A negative voltage between -3 and $-6 \mathrm{~V}$ was applied to the sample while it was gradually immersed into the solution. $\mathrm{H}_{2}$ bubbles form at the $h$-BN/Rh interface and delaminate the $h$-BN/TOA/PMMA film from the 


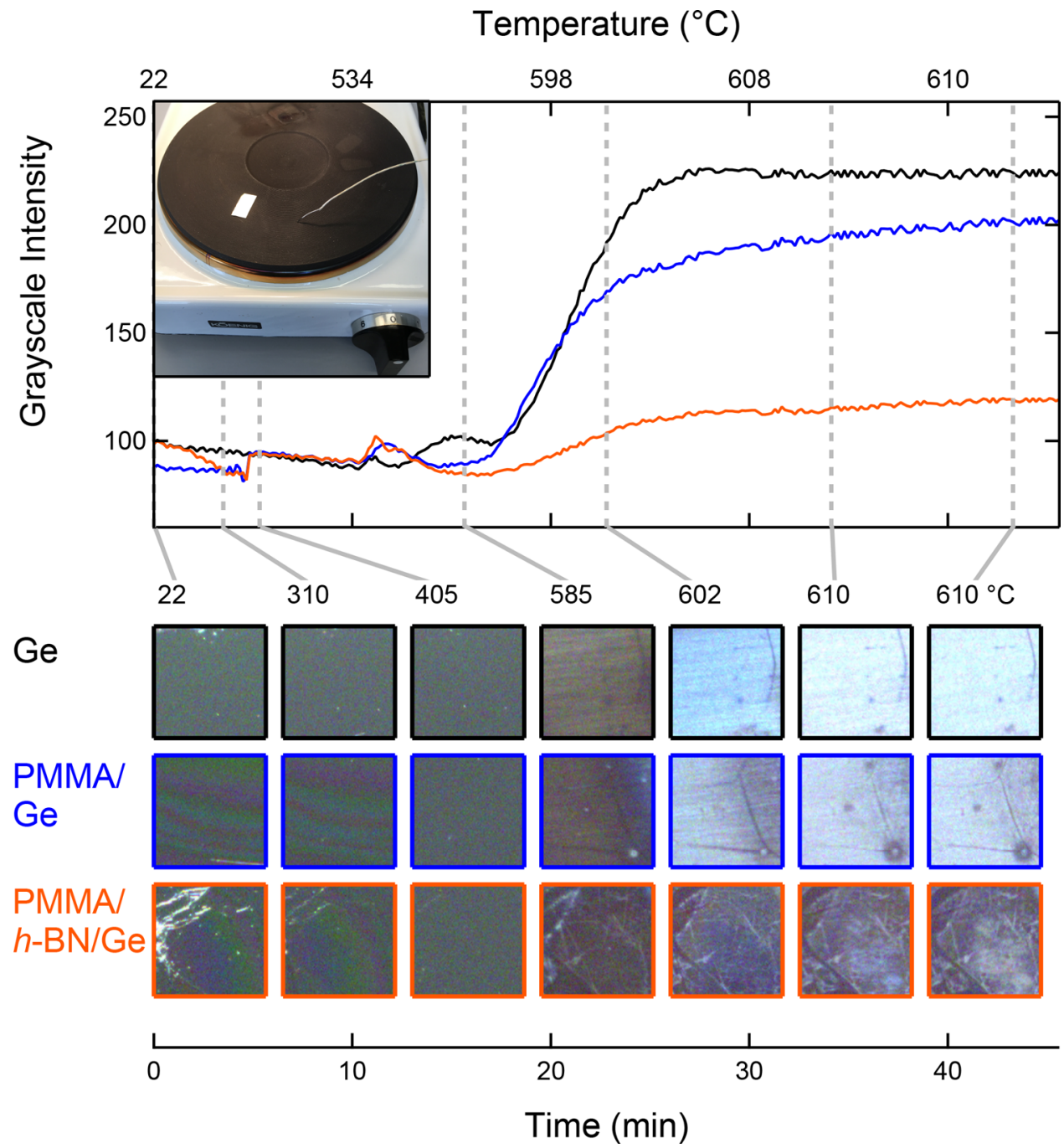

Figure 4. Large-scale high-temperature protection of Ge against oxidation by transferred $h$-BN. The inset shows the heating plate with a piece of Ge wafer on top. PMMA and transferred PMMA/h-BN are placed on a Ge(111) surface. Top panel: grayscale intensities of the camera images of Ge (black), PMMA/Ge (blue), and PMMA/ $h$-BN/Ge (red) as a function of sample temperature and annealing time. The process involves three stages: (1) PMMA desorption at $300-350{ }^{\circ} \mathrm{C}$. (2) At $\sim 405{ }^{\circ} \mathrm{C}$, the three samples show the same color. (3) At $585{ }^{\circ} \mathrm{C}$, bare Ge and PMMA/Ge (after PMMA removal) start oxidizing, and the colors of the surfaces turn bright blue (Ge bulk oxide formation), while PMMA/ $h$-BN/Ge stays dark even after $10 \mathrm{~min}$ at $610^{\circ} \mathrm{C}$. Bottom frame panel: Surface color changes of the three regions ( 7 frames each) vs time and for temperatures from room temperature up to $610^{\circ} \mathrm{C}$. All squares represent $2 \mathrm{~mm} \times 2 \mathrm{~mm}$. The color codes of the frame-boarders are the same as the traces in the top panel.

substrate. The delaminated $h$-BN/TOA/PMMA film was then rinsed in ultrapure water (Milli-Q Advantage A10) and transferred on a clean $80 \mathrm{~nm} \mathrm{SiO}_{2} / \mathrm{Si}$ substrate. In the next step, the PMMA was removed via a sequence of acetone/ ethanol baths at $370 \mathrm{~K}$ and gradual anneal in air up to $600 \mathrm{~K}$ for $3 \mathrm{~h}$. Two types of TEM grids were used: (1) Cu quantifoil with an empty hole size of $1000 \mathrm{~nm}$ and interval distance of holes of $800 \mathrm{~nm}$ (Figure 5a) and (2) $\mathrm{SiN}_{x}$ with a thickness of $20 \mathrm{~nm}$ and a single hole with $50 \mathrm{~nm}$ in diameter in the middle (Figure $5 \mathrm{~d}$ ). The TEM grids were coated with $25 \mathrm{~nm} \mathrm{Pt}$ prior to the transferred-layer deposition. The Pt acts as a catalyst that reduces the PMMA disintegration temperature and improves the PMMA removal efficiency. ${ }^{36}$ After transfer, the PMMA/ $\mathrm{TOA} / h-\mathrm{BN} /$ grids were slowly heated in air to $600 \mathrm{~K}$ for $5 \mathrm{~h}$ to remove PMMA/TOA. The $\mathrm{Rh}(111)$ film substrate was then put back to the UHV system and heated up to $800 \mathrm{~K}$ before XPS and low-energy electron diffraction (LEED) measurements.
Sample Characterizations. All sample characterizations were carried out at room temperature. The scanning tunneling microscopy (STM) measurements were accomplished with an Omicron variable temperature STM (VT-STM) with electrochemically etched tungsten tips. All STM images were taken in constant-current mode.

Optical images were acquired using a Leica DMV2500 stereomicroscope with a maximum magnification of $2500 \times$. Sequential frame acquisition was performed using procedures written with the Leica Self-Development Kit package, and the frames were subsequently stitched using the Fiji imageprocessing package.

XPS, X-ray photoelectron diffraction (XPD), and LEED measurements were carried out on a user-modified Vacuum Generators ESCALAB $220^{37}$ with a Mg K $\alpha$ lab source at an energy of $\hbar \omega=1253.6 \mathrm{eV}$. X-ray photoemission electron microscopy (X-PEEM) images were recorded at the boron Kedge at the Surface/Interface Microscopy (SIM) beamline of the Swiss Light Source (SLS). 

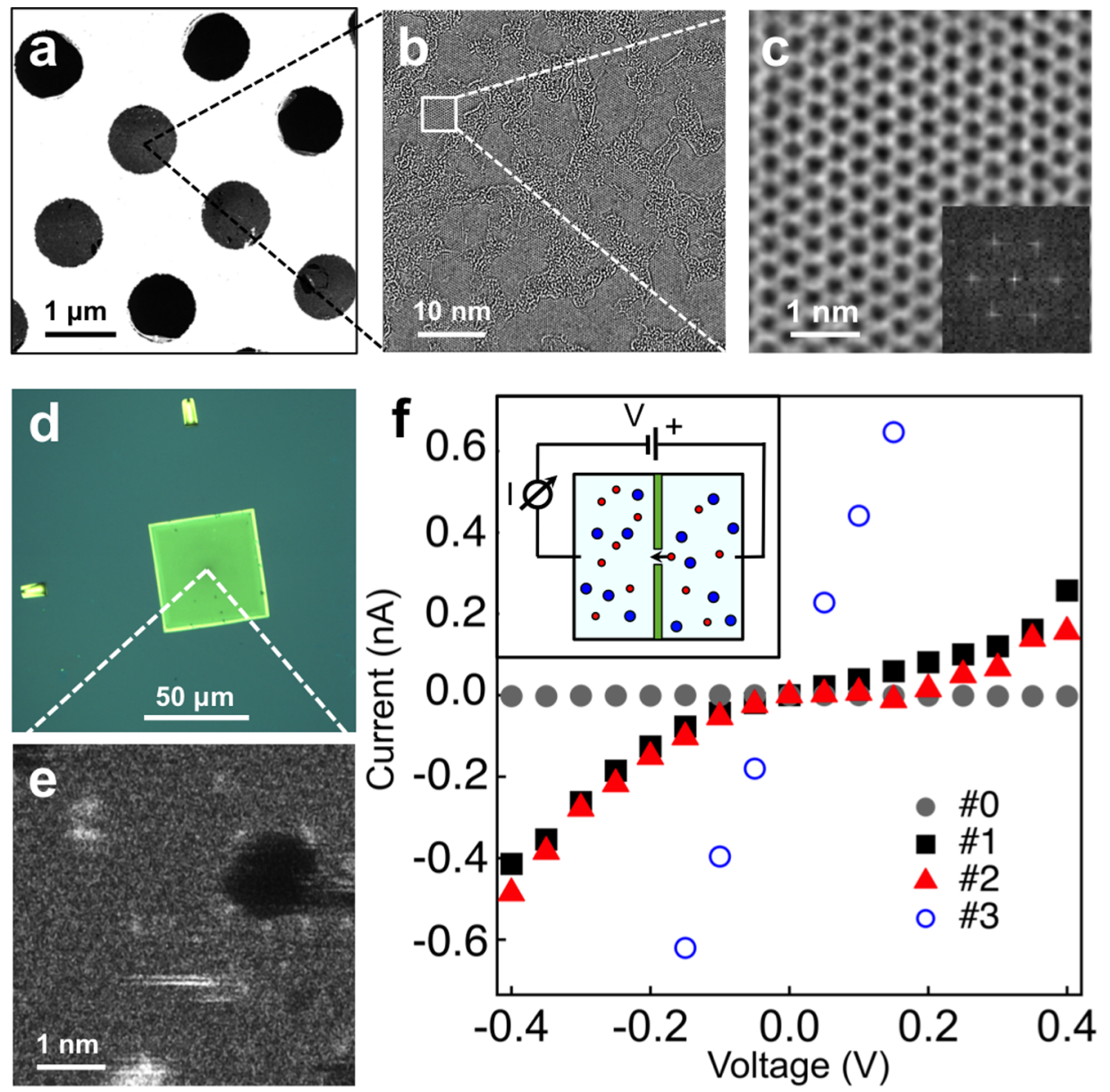

Figure 5. Freestanding $h$-BN membranes. (a) Dark-field TEM image of a large-area $h$-BN membrane suspended over a Cu quantifoil TEM grid. (b) Zoomed-in TEM image displays freestanding single-layer $h$-BN, with some transfer-related residues. (c) Further zoomed-in high-resolution (HR)TEM image: Single-crystalline BN honeycomb lattice with atomic resolution. Inset: Fast Fourier transform (FFT) of panel c, confirming the crystalline structure with hexagonal symmetry. (d) Optical microscopy image of a $h$-BN membrane with $2 \mathrm{~nm}$ voids on a $20 \mathrm{~nm} \mathrm{SiN}_{x}$ membrane with $50 \mathrm{~nm}$ hole in the middle. (e) HR-STEM image of a $h$-BN membrane with a $2 \mathrm{~nm}$ void. (f) Current vs voltage characteristic of 4 different $v$-BN membrane samples in $10 \mathrm{mM} \mathrm{KCl}$ solutions at $\mathrm{pH} 8$. The inset shows the sketch of the setup. All TEM and STEM images were taken at $80 \mathrm{keV}$ electron energy and with the sample at room temperature.

Raman measurements were performed with a Thermo Scientific DXR 2 Raman Microscope equipped with CCR1000 Linkam at $455 \mathrm{~nm}$ excitation wavelength. Scanning electron microscopy in Figure S2 were measured using a Zeiss Supra 50 VP field-emission SEM equipped with secondary, backscattered electron and EDX detectors at energies between $2-2.5 \mathrm{keV}$.

TEM and STEM images were measured on an aberrationcorrected (with double Cs corrector) FEI Titan Themis TEM 60-300 operated at an $80 \mathrm{kV}$ acceleration voltage. For HRTEM imaging, a spherical aberration (Cs) of about -20 $\mu \mathrm{m}$ was applied, combined with a monochromatic incident illumination to reduce the effects of chromatic aberration, for an optical setup capable of $1 \AA$ resolution or better. Images are shown with focus adjusted to give a white atom contrast, ${ }^{38}$ which, when imaging a clean uncontaminated monolayer, allows the phase-contrast HRTEM image to be directly interpreted as atomic positions. (S)TEM image processing: Raw TEM images were filtered by using a bandpass filter between 1 and 100 pixels. These filtered images were then blurred out using a Gaussian blur function with a diameter of 1.2 pixels to enhance visual inspections.
Nanofluidic transport experiments were performed using the setup described previously. ${ }^{31}$ After mounting in the chamber, chips were wet with $\mathrm{H}_{2} \mathrm{O}$ /ethanol (1:1) solution for $30 \mathrm{~min}$. Nanofluidic measurements were performed by taking the current-voltage response ( $I-V$ characteristics, respectively) of the nanovoid in salt solutions of potassium chloride $(\mathrm{KCl}$, Sigma-Aldrich) using an Axopatch 200B patch-clamp amplifier (Molecular Devices, Inc. Sunnyvale, CA) with voltage range from -1.0 to $1.0 \mathrm{~V}$.

\section{ASSOCIATED CONTENT}

\section{S Supporting Information}

The Supporting Information is available free of charge on the ACS Publications website at DOI: 10.1021/acs.nanolett.7b04752.

Transfer-methods comparison, reusability of substrates, characterization details, ion conductivity model, and supporting data. (PDF)

\section{AUTHOR INFORMATION}

\section{Corresponding Author}

*E-mail: huanyao.cun@epfl.ch. 


\section{ORCID}

Huanyao Cun: 0000-0002-5225-9861

Benjamin Probst: 0000-0001-8850-9685

Ke Liu: 0000-0002-1976-2297

Duncan T. L. Alexander: 0000-0003-4350-8587

Gerson Mette: 0000-0001-7561-3264

Aleksandra Radenovic: 0000-0001-8194-2785

\section{Notes}

The authors declare no competing financial interest.

\section{ACKNOWLEDGMENTS}

Financial support by the Swiss National Science Foundation and by the EC under the Graphene Flagship (contract no. CNECT-ICT-604391) is gratefully acknowledged. We gratefully thank Prof. Dr. Wlodek Strupinski and Dr. Aleksandra Krajewska from the Institute of Electronic Materials Technology in Warsaw for introducing the electrochemical graphene transfer from Cu foil. We thank Dr. Qasim Imtiaz, Manouchehr Nadjafi, and Dr. Miroslav Haluska from ETH Zürich for introducing the Raman instruments. Part of this work was performed at the Surface/Interface Microscopy (SIM) beamline of the Swiss Light Source, Paul Scherrer Institut (PSI), Villigen, Switzerland. We thank Marianna Lorenzo and Dr. Jean-Nicholas Longchamp for $\mathrm{Pt}$ coating and fruitful discussions, Anja Weber (PSI) for providing the $\mathrm{SiO}_{2} / \mathrm{Si}$ substrates, and the Centre Interdisciplinaire de Microscopie Electronique (CIME) at the EPFL Lausanne and the Center for Microscopy and Image Analysis of the University of Zurich (Dr. Andres Käch) for access to electron microscopes.

\section{REFERENCES}

(1) Geim, A. K.; Grigorieva, I. V. Van der Waals heterostructures. Nature 2013, 499, 419-425.

(2) Mak, K. F.; Lee, C.; Hone, J.; Shan, J.; Heinz, T. F. Atomically thin $\mathrm{MoS}_{2}$ : A new direct-gap semiconductor. Phys. Rev. Lett. 2010, 105, 136805.

(3) Siria, A.; Poncharal, P.; Biance, A.; Fulcrand, R.; Blase, X.; Purcell, S. T.; Bocquet, L. Giant osmotic energy conversion measured in a single transmembrane boron nitride nanotube. Nature 2013, 494, $455-458$.

(4) Mertens, S.; Hemmi, A.; Muff, S.; Gröning, O.; De Feyter, S.; Osterwalder, J.; Greber, T. Switching stiction and adhesion of a liquid on a solid. Nature 2016, 534, 676-679.

(5) Feng, J.; Graf, M.; Liu, K.; Ovchinnikov, D.; Dumcenco, D.; Heiranian, M.; Nandigana, V.; Aluru, N. R.; Kis, A.; Radenovic, A. Single-layer $\mathrm{MoS}_{2}$ nanopores as nanopower generators. Nature 2016, 536, 197-200.

(6) Dean, C. R.; Young, A. F.; Meric, I.; Lee, C.; Wang, L.; Sorgenfrei, S.; Watanabe, K.; Taniguchi, T.; Kim, P.; Shepard, K. L.; Hone, J. Boron nitride substrates for high-quality graphene electronics. Nat. Nanotechnol. 2010, 5, 722-726.

(7) Gao, Y.; Ren, W.; Ma, T.; Liu, Z.; Zhang, Y.; Liu, W.; Ma, L.; Ma, X.; Cheng, H.-M. Repeated and controlled growth of monolayer, bilayer and few-Layer hexagonal boron nitride on Pt foils. ACS Nano 2013, 7, 5199-5206.

(8) Jang, A.-R.; Hong, S.; Hyun, C.; Yoon, S. I.; Kim, G.; Jeong, H. Y.; Shin, T. J.; Park, S. O.; Wong, K.; Kwak, S. K.; Park, N.; Yu, K.; Choi, E.; Mishchenko, A.; Withers, F.; Novoselov, K. S.; Lim, H.; Shin, $\mathrm{H}$. K. Wafer-scale and wrinkle-free epitaxial growth of single-orientated multilayer hexagonal boron nitride on sapphire. Nano Lett. 2016, 16, 3360-3366.

(9) Oh, H.; Jo, J.; Tchoe, Y.; Yoon, H.; Hwi Lee, H.; Kim, S.-S.; Kim, M.; Sohn, B.-H.; Yi, G.-C. Centimeter-sized epitaxial h-BN films. NPG Asia Mater. 2016, 8, e330.
(10) Gsell, S.; Fischer, M.; Schreck, M.; Stritzker, B. Epitaxial films of metals from the platinum group ( $\mathrm{Ir}, \mathrm{Rh}, \mathrm{Pt}$ and $\mathrm{Ru}$ ) on YSZ-buffered Si(111). J. Cryst. Growth 2009, 311, 3731-3736.

(11) Hemmi, A.; Bernard, C.; Cun, H. Y.; Roth, S.; Klöckner, M.; Kälin, T.; Weinl, M.; Gsell, S.; Schreck, M.; Osterwalder, J.; Greber, T. High quality single atomic layer deposition of hexagonal boron nitride on single crystalline $\mathrm{Rh}(111)$ four-inch wafers. Rev. Sci. Instrum. 2014, 85,035101

(12) Corso, M.; Auwärter, W.; Muntwiler, M.; Tamai, A.; Greber, T.; Osterwalder, J. Boron nitride nanomesh. Science 2004, 35, 217-220.

(13) Berner, S.; Corso, M.; Widmer, R.; Groening, O.; Laskowski, R.; Blaha, P.; Schwarz, K.; Goriachko, A.; Over, H.; Gsell, S.; Schreck, M.; Sachdev, H.; Greber, T.; Osterwalder, J. Boron nitride nanomesh: Functionality from a corrugated monolayer. Angew. Chem., Int. Ed. 2007, 46, 5115-5119.

(14) Gomez Díaz, J.; Ding, Y.; Koitz, R.; Seitsonen, A. P.; Iannuzzi, M.; Hutter, J. Hexagonal boron nitride on transition metal surfaces. Theor. Chem. Acc. 2013, 132, 1350-1366.

(15) Widmer, R.; Berner, S.; Gröning, O.; Brugger, T.; Osterwalder, J.; Greber, T. Electrolytic in situ STM investigation of h-BNNanomesh. Electrochem. Commun. 2007, 9, 2484-2488.

(16) Brugger, T.; Ma, H.; Iannuzzi, M.; Berner, S.; Winkler, A.; Hutter, J.; Osterwalder, J.; Greber, T. Nanotexture switching of singlelayer hexagonal boron nitride on rhodium by intercalation of hydrogen atoms. Angew. Chem., Int. Ed. 2010, 49, 6120-6124.

(17) Cun, H. Y.; Iannuzzi, M.; Hemmi, A.; Roth, S.; Osterwalder, J.; Greber, T. Immobilizing individual atoms beneath a corrugated single layer of boron nitride. Nano Lett. 2013, 13, 2098-2103.

(18) Cun, H. Y.; Iannuzzi, M.; Hemmi, A.; Osterwalder, J.; Greber, T. Two-Nanometer voids in single-layer hexagonal boron nitride: Formation via the "can-opener" effect and annihilation by self-healing. ACS Nano 2014, 8, 7423-7431.

(19) Cun, H. Y.; Iannuzzi, M.; Hemmi, A.; Osterwalder, J.; Greber, T. Implantation length and thermal stability of interstitial Ar stoms in boron nitride nanotents. ACS Nano 2014, 8, 1014-1021.

(20) Koefoed, L.; Kongsfelt, M.; Ulstrup, S.; Čabo, A. G.; Cassidy, A.; Whelan, P. R.; Bianchi, M.; Dendzik, M.; Pizzocchero, F.; Jørgensen, B.; Bøggild, P.; Hornekaer, L.; Hofmann, P.; Pedersen, S. U.; Daasbjerg, K. Facile electrochemical transfer of large-area single crystal epitaxial graphene from $\operatorname{Ir}(111)$. J. Phys. D: Appl. Phys. 2015, 48, 115306.

(21) Wang, Y.; Zheng, Y.; Xu, X.; Dubuisson, E.; Bao, Q.; Lu, J.; Loh, K. P. Electrochemical delamination of CVD-Grown graphene film: Toward the recyclable use of copper catalyst. ACS Nano 2011, 5, 9927-9933.

(22) Ciuk, T.; Pasternak, I.; Krajewska, A.; Sobieski, J.; Caban, P.; Szmidt, J.; Strupinski, W. Properties of chemical vapor deposition graphene transferred by high-speed electrochemical delamination. $J$. Phys. Chem. C 2013, 117, 20833-20837.

(23) Gorbachev, R. V.; Riaz, I.; Nair, R. R.; Jalil, R.; Britnell, L.; Belle, B. D.; Hill, E. W.; Novoselov, K. S.; Watanabe, K.; Taniguchi, T.; Geim, A. K.; Blake, P. Hunting for monolayer boron nitride: Optical and Raman signatures. Small 2011, 7, 465-468.

(24) Auwärter, W.; Kreutz, T.; Greber, T.; Osterwalder, J. XPD and STM investigation of hexagonal boron nitride on $\mathrm{Ni}(111)$. Surf. Sci. 1999, 429, 229-236.

(25) Roth, S.; Greber, T.; Osterwalder, J. Some Like It Flat: Decoupled h-BN Monolayer Substrates for Aligned Graphene Growth. ACS Nano 2016, 10, 11187-11195.

(26) Sahari, S. K.; Ohta, A.; Matsui, M.; Mishima, K.; Murakami, H.; Higashi, S.; Miyazaki, S. Kinetics of thermally oxidation of $\mathrm{Ge}(100)$ surface. J. Phys.: Confer. Seri. 2013, 417, 012014.

(27) Krivanek, O. L.; Chisholm, M. F.; Nicolosi, V.; Pennycook, T. J.; Corbin, G. J.; Dellby, N.; Murfitt, M. F.; Own, C. S.; Szilagyi, Z. S.; Oxley, M. P.; Pantelides, S. T.; Pennycook, S. J. Atom-by-atom structural and chemical analysis by annular dark-field electron microscopy. Nature 2010, 464, 571-574. 
(28) Meyer, J. C.; Chuvilin, A.; Algara-Siller, G.; Biskupek, J.; Kaiser, $\mathrm{U}$. Selective sputtering and atomic resolution imaging of atomically thin boron nitride membranes. Nano Lett. 2009, 9, 2683-2689.

(29) Kotakoski, J.; Jin, C. H.; Lehtinen, O.; Suenaga, K.; Krasheninnikov, A. V. Electron knock-on damage in hexagonal boron nitride monolayers. Phys. Rev. B: Condens. Matter Mater. Phys. 2010, 82, 113404.

(30) O’Hern, S. C.; Boutilier, M. S. H.; Idrobo, J.-C.; Song, Y.; Kong, J.; Laoui, T.; Atieh, M.; Karnik, R. Selective ionic transport through tunable subnanometer pores in single-layer graphene membranes. Nano Lett. 2014, 14, 1234-1241.

(31) Liu, K.; Feng, J.; Kis, A.; Radenovic, A. Atomically thin molybdenum disulfide nanopores with high sensitivity for DNA translocation. ACS Nano 2014, 8, 2504-2511.

(32) Hall, J. E. Access resistance of a small circular pore. J. Gen. Physiol. 1975, 66, 531-532.

(33) Kowalczyk, S. W.; Grosberg, A. Y.; Rabin, Y.; Dekker, C. Modeling the conductance and DNA blockade of solid-state nanopores. Nanotechnology 2011, 22, 315101.

(34) Lee, C.; Joly, L.; Siria, A.; Biance, A.-L.; Fulcrand, R.; Bocquet, L. Large apparent electric size of solid-state nanopores due to spatially extended surface conduction. Nano Lett. 2012, 12, 4037-4044.

(35) Hu, S.; Lozada-Hidalgo, M.; Wang, F. C.; Mishchenko, A.; Schedin, F.; Nair, P. R.; Hill, E. W.; Boukhvalov, D. W.; Katsnelson, M. I.; Dryfe, R. A. W.; Grigorieva, I. V.; Wu, H. A.; Geim, A. K. Proton transport through one-atom-thick crystals. Nature 2014, 516, 227230.

(36) Longchamp, J. N.; Escher, C.; Fink, H. W. Ultraclean freestanding graphene by platinum-metal catalysis. J. Vac. Sci. Technol, B: Nanotechnol. Microelectron.: Mater., Process., Meas., Phenom. 2013, 31, 020605 .

(37) Greber, T.; Raetzo, O.; Kreutz, T.; Schwaller, P.; Deichmann, W.; Wetli, E.; Osterwalder, J. A photoelectron spectrometer for kspace mapping above the Fermi level. Rev. Sci. Instrum. 1997, 68, $4549-4554$

(38) Ryu, G. H.; Park, H. J.; Ryou, J.; Park, J.; Lee, J.; Kim, G.; Shin, H. S.; Bielawski, C. W.; Ruoff, R. S.; Hong, S.; Lee, Z. Atomic-scale dynamics of triangular hole growth in monolayer hexagonal boron nitride under electron irradiation. Nanoscale 2015, 7, 10600-10605. 